Available online at GSC Online Press Directory

GSC Advanced Research and Reviews e-ISSN: 2582-4597, CODEN (USA): GARRC2

Journal homepage: https://www.gsconlinepress.com/journals/gscarr

(CASE STUdy)

\title{
Severe dengue fever: A case report
}

\author{
Mishra Ved Prakash ${ }^{1,}{ }^{*}$, Twanabasu Suraj ${ }^{2}$, Kusma Susan ${ }^{3}$ and Thapa Kajol ${ }^{4}$ \\ ${ }^{1}$ Lecturer, Department of Microbiology, Kathmandu School of Medical Technology, CTEVT, Ratopul, Kathmandu, Nepal. \\ ${ }^{2}$ Medical Laboratory Technologist, Department of Pathology, Vayodha Hospitals Pvt. Ltd., Balkhu, Kathmandu, Nepal. \\ ${ }^{3}$ Medical Laboratory Technologist, Department of Pathology, Lalupate Medical Center Pvt. Ltd., Gaushala, Kathmandu, \\ Nepal. \\ ${ }^{4}$ Coordinator, Department of Medical Laboratory Technology, Kathmandu School of Medical Technology, CTEVT, Ratopul, \\ Kathmandu, Nepal.
}

Publication history: Received on 15 June 2020; revised on 23 June 2020; accepted on 25 June 2020

Article DOI: https://doi.org/10.30574/gscarr.2020.3.3.0051

\begin{abstract}
Dengue is a mosquito-borne viral infection with many clinically diverse spectrums of presentations. Although most of the cases are diagnosed clinically, even a simple full blood count (FBC) can assist to confirming this often deceiving infection, which allows the initiation of potentially lifesaving treatment. In this article, two cases of dengue are mentioned, one of which has a high hematocrit and hemoglobin value, which can lead to multiple organ failure and disseminated intravascular coagulation (DIC) and another one added with several atypical clinical manifestation. Thrombocytopenia and leukopenia with positive dengue-NS1 antigen were seen in both cases. Both cases were treated with supportive care and intravenous fluid to improve symptoms. With such complications, there is a need to further examine these cases further in detail.
\end{abstract}

Keywords: Dengue fever; Capillary leakage; Hematocrit; Ascites

\section{Introduction}

Dengue (a mosquito borne viral infection) is a pathological condition caused by single positive- stranded RNA virus of the family Flaviviridae called Dengue Virus (DENV). DENV is grouped into five serotypes, and these serotypes are antigenically distinct, although closely related $\left(\mathrm{DENV}_{1}, \mathrm{DENV}_{2}, \mathrm{DENV}_{3}, \mathrm{DENV}_{4}\right.$ and $\left.\mathrm{DENV}_{5}\right)$ with the fifth serotype recently discovered in late 2013 [1,2]. Dengue virus is transmitted by female mosquitoes mainly of the species Aedes aegypti and, to a lesser extent, Ae. Albopictus [3]. Dengue is widespread throughout the tropics, with local variations in risk influenced by rainfall, temperature, relative humidity and unplanned rapid urbanization [3]. Global warming has significantly increased the spread of the disease.

The WHO has identified several criteria for dengue diagnosis based on symptoms and laboratory findings; people living in endemic areas or those who travel to such areas with the likelihood of dengue, manifesting two of the following can be suspected of this fever:

- Nausea, vomiting, rash, pain, positive tourniquet test, leukopenia

- Warning signs including abdominal pain and tenderness

- Persistent vomiting and fluid retention

- Mucosal bleeding, lethargy and irritability

- Enlarged liver more than $2 \mathrm{~cm}$

- Elevated hematocrit with a rapid decrease in platelets $[4,5]$.

\footnotetext{
${ }^{*}$ Corresponding author: Mishra Ved Prakash
} 
There is no any specific treatment devised for this disease; rather, the treatment is supportive; however, the invention of the first dengue vaccine Dengvaxia (CYD-TDV) has brought hope. Tested in clinical trials, vaccination was proven to be effective at preventing severe dengue fever and also successfully reduced admissions to the hospital by $80 \%$ [6, 7]. However, cost and actual effectiveness when used in real time have shown mixed results [7]. Mass community education, vector control measures and awareness about transmission of disease can play a vital role in preventing and safeguarding oneself in endemic areas.

\section{Case Presentation}

\subsubsection{Case 1}

A 23-year-old man presented with a 6-day history of intermittent fever, bodily pain, nausea, lethargy and vomiting. He suffered occasional diarrhea and oral intake was reduced along with appetite. He also suffered from retro-orbital headache and pain in the joints. Blood pressure of $110 / 80 \mathrm{mmHg}$, pulse rate of 100 beats per minute, a temperature of $100.1^{\mathrm{o}} \mathrm{F}$ and respiratory rate of 20 breaths per minute without any remarkable abdominal and respiratory findings. His hydration status was fair and his urine output was normal. His last paracetamol intake was 19 hours before admission.

Laboratory findings are presented in Table.1. Results of chest X-ray and ultrasonography (USG) were normal along with electrocardiogram (ECG). A Rapid immunological dengue cartridge test showed Dengue-NS1 positive; Dengue- IgM and IgG were negative. He was diagnosed with severe dengue in the defervescence stage and was referred for admission. While admitted, he was given intravenous fluids and supportive care and was discharged after 5 days when all his clinical signs and symptoms were subsidized and all laboratory tests were normal. The patient was requested to follow up after a week. His hematocrit (PCV) was $44 \mathrm{~g} / \mathrm{dL}$, platelet count of 250,000 cells $/ \mathrm{mm}^{3}$ and WBC count of 7500 cells $/ \mathrm{mm}^{3}$ during the follow up.

Table 1 Hematological and biochemical parameters during the hospital stay of the patient

\begin{tabular}{|c|c|c|c|c|c|}
\hline $\begin{array}{l}\text { Clinical and laboratory } \\
\text { parameters }\end{array}$ & $\begin{array}{l}\text { At the time of } \\
\text { admission }\end{array}$ & $2^{\text {nd }}$ day & $3^{\text {rd day }}$ & $4^{\text {th }}$ day & $5^{\text {th }}$ day \\
\hline $\begin{array}{l}\text { Total leukocyte count }\left(\begin{array}{lll}\mathrm{x} & 10^{3} \\
\text { cells } / \mathrm{mm}^{3} \text { ) }\end{array}\right.\end{array}$ & 2.1 & 1.8 & 2.5 & 3.7 & 4.8 \\
\hline Platelet count $\left(\right.$ x $10^{3}$ cells $/ \mathrm{mm}^{3}$ ) & 80 & 84 & 121 & 107 & 151 \\
\hline Hemoglobin (g/dL) & 15.2 & 15 & 14.9 & 14.5 & 14 \\
\hline Hematocrit \% & $53 \%$ & $50 \%$ & $48 \%$ & $46.20 \%$ & $45 \%$ \\
\hline SGOT (IU/L) & 87 & 50 & 32 & - & - \\
\hline SGPT (IU/L) & 59 & 39 & 30 & - & - \\
\hline Prothrombin time (seconds) & 13.8 & - & - & - & - \\
\hline INR & 1 & - & - & - & - \\
\hline
\end{tabular}

*SGOT- Serum glutamic oxaloacetic transaminase, *SGPT- Serum Glutamic Pyruvic Transaminase

\subsubsection{Case 2}

An 18-year-old woman presented with a history of fever for 3 days along with chills and rigor. She suffered pain in the lower abdomen, severe aching type since 2 days. Vomiting with multiple episodes containing undigested food particles, non-bilious and not mixed with blood. She suffered a burning micturition. There was no PV discharge. Physical examinations revealed a temperature of $101.2^{\circ} \mathrm{F}$ with tachycardia up to 110 beats/min, respiratory rate of 22 breaths/min and were hypotensive with a blood pressure of 70/50 $\mathrm{mmHg}$. Soft tenderness was seen over the suprapubic region.

She was managed with fluid and antibiotics with a provisional diagnosis of complicated UTI (Urinary Tract Infection). Ultrasound abdomen showed features of cystitis; however, urine routine examination was not suggestive of cystitis. In search of the source, rapid dengue test was sent, which was positive for NS1. 

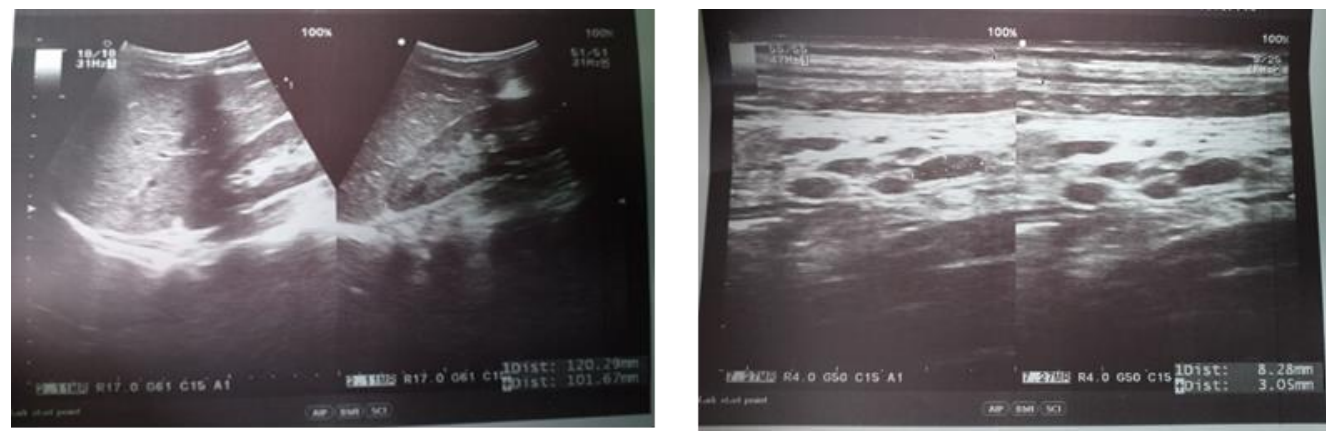

Figure Ultrasonography of the abdomen

Table 2 Hematological and biochemical parameters during the hospital stay of the patient

\begin{tabular}{|c|c|c|c|c|}
\hline Clinical and laboratory parameters & At the time of admission & $2^{\text {nd }}$ day & $3^{\text {rd }}$ day & $4^{\text {th }}$ day \\
\hline Total leukocyte count $\left(\mathrm{x} 10^{3}\right.$ cells $\left./ \mathrm{mm}^{3}\right)$ & 3.1 & 3.9 & 4.8 & 5.7 \\
\hline Hemoglobin $(\mathrm{g} / \mathrm{dL})$ & 10.8 & 11.1 & 11.5 & 11.9 \\
\hline Hematocrit \% & 32 & 33.5 & 34.1 & 35 \\
\hline Platelet count $\left(\right.$ x $10^{3}$ cells $/ \mathrm{mm}^{3}$ ) & 105 & 134 & 158 & 190 \\
\hline serum urea $(\mathrm{mg} / \mathrm{dL})$ & 8 & 10.2 & 13.6 & 19.8 \\
\hline serum creatinine $(\mathrm{mg} / \mathrm{dL})$ & 0.8 & 0.9 & 0.8 & 0.9 \\
\hline serum sodium (mEq/L) & 137 & 140 & 139 & 138 \\
\hline serum potassium $(\mathrm{mEq} / \mathrm{L})$ & 4.2 & 4.3 & 3.9 & 3.8 \\
\hline SGOT (IU/L) & 11 & 13 & 17 & 27 \\
\hline SGPT (IU/L) & 27 & 26 & 31 & 35 \\
\hline
\end{tabular}

*SGOT- Serum glutamic oxaloacetic transaminase, *SGPT- Serum Glutamic Pyruvic Transaminase

\section{Discussion}

Dengue is a common mosquito borne viral disease that seen mainly in the Asia-Pacific region [8, 9]. It can present with a diverse clinical spectrum ranging from asymptomatic infection or simple undifferentiated fever to DHF with multiorgan failure [9] and dengue shock syndrome. The disease can manifest as three phases; namely febrile, afebrile (defervescence) and recovery (convalescence) phase. Of these 3 stages, the most critical stage is afebrile stage, which can be deceiving to the unexperienced physician because the patient may have massive leaking of the fluids and internal bleeding, but look deceivingly well [7]. This can be indicated by a hematocrit level of more than $20 \%$ of the baseline level [7]. Therefore, monitoring and normalizing hematocrit and not platelets should be the main therapeutic goal to prevent severe complications of dengue fever, such as massive bleeding and shock [7]. The first case presented with a similar mentioned scenario in which action was taken promptly, which could have devastating effects on the patient if any delay occurred.

Looking at the mentioned two cases, treatment of dengue fever can be difficult and its diagnosis can be misleading. The first case complicated a significantly abnormal blood count with a high hematocrit of $53 \%$ and a low platelet of $80 \times 10^{3}$ cells $/ \mathrm{mm}^{3}$. The presence of high hematocrit, especially more than $20 \%$ higher than the baseline level, should be taken seriously as it indicates capillary leakage [7]. Capillary leakage, if not contained early, may lead to multiple organ failure and DIC, which has a high mortality rate $[7,10]$. Hence, even though physicians are encouraged to treat their patients mainly on clinical judgement, it is equally important to do basic full blood count (FBC) not to miss this important diagnosis [7].

The second case presented a slightly different clinical scenario where, unlike first case, hemoglobin and hematocrit were fairly low. The Patient suffered hypotension with severe abdominal pain entangled with many atypical manifestations. Hence, it is not always imperative that all dengue infections manifest the same clinical symptoms and physicians must be skilled enough to deal with any symptoms of dengue, as dengue can represent itself in any manner. 
Therefore, for severe dengue, medical care by physicians and nurses experienced with the effects and progression of the disease can save lives by decreasing the mortality rate from more than $20 \%$ to less than $1 \%$ [3]. Maintenance of the patient's body fluid volume is critical to severe dengue care [3].

\section{Conclusion}

Dengue is a potentially life-threatening illness unless promptly diagnosed and properly treated. Clinical presentation and thrombocytopenia with high hematocrit is the most common pointer to the causes. However, the presentation can be deceiving; hence, a high index of suspicion is the key to early diagnosis.

\section{Compliance with ethical standards}

\section{Acknowledgments}

The authors are grateful to all those who helped and supported in preparing this case report.

\section{Disclosure of conflict of interest}

The authors declare no conflicts of interest.

\section{Statement of informed consent}

Informed consent was obtained from all individual participants included in the study.

\section{References}

[1] Mustafa MS, Rasotgi V, Jain S and Gupta V. (2015). Discovery of fifth serotype of dengue virus (DENV-5): a new public health dilemma in dengue control. Med J Armed Forces India, 71(1), 67-70.

[2] Norazharuddin H and Lai NS. (2018). Roles and prospects of dengue virus non-structural proteins as antiviraltargets: an easy digest. Malays J Med Sci, 25(5), 6-15.

[3] WHO. (2 March, 2020). Dengue and severe dengue. WHO website.

[4] World Health Organization. (2009). Dengue guidelines for diagnosis, treatment, prevention and Control: new edition. World Health Organization.

[5] Ebrahimi M, Abadi A, Bashizadeh-Fakhar H and Fahimi E. (2016). Dengue Fever in Iran: A Case Report. Zahedan J Res Med Sci, 18(12), e9953.

[6] JP Cunha, "Dengue fever", MedicineNet.com, 2017. [Online].

[7] NK Devaraj. (2018). Case reports: Two atypical cases of severe dengue fever. Rwanda Medical Journal, 75(3).

[8] Halstead SB. Dengue. (2002). Curr Opin Infect Dis, 15(5), 471-6.

[9] Dalugama and Gawarammana. (2017). Dengue hemorrhagic fever complicated with acute liver failure: a case report. Journal of Medical Case Reports, 11, 341.

[10] P Amin, Ö Acicbe, J Hidalgo, JI Jiménez, T Baker and GA Richards. (2018). Dengue fever: report from the task force on tropical diseases by the world federation of societies of intensive and critical care medicine. Journal of Critical care, 43, 346-51.

\section{How to cite this article}

Mishra VP, Twanabasu S, Kusma S and Thapa K. (2020). Severe dengue fever: A case report. GSC Advanced Research and Reviews, 3(3), 79-82. 